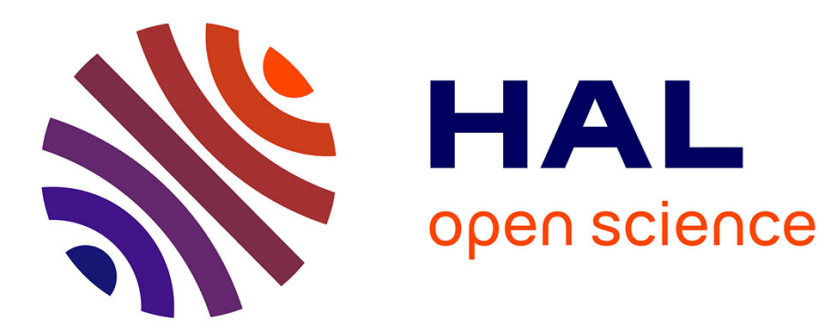

\title{
Résistance du substrat de carbone au silicium fondu dans le procédé RAD
}

\author{
J. Goma, A. Oberlin, E. Kerrand, C. Belouet
}

\section{To cite this version:}

J. Goma, A. Oberlin, E. Kerrand, C. Belouet. Résistance du substrat de carbone au silicium fondu dans le procédé RAD. Revue de Physique Appliquée, 1984, 19 (4), pp.297-306. 10.1051/rphysap:01984001904029700 . jpa-00245196

\section{HAL Id: jpa-00245196 https://hal.science/jpa-00245196}

Submitted on 1 Jan 1984

HAL is a multi-disciplinary open access archive for the deposit and dissemination of scientific research documents, whether they are published or not. The documents may come from teaching and research institutions in France or abroad, or from public or private research centers.
L'archive ouverte pluridisciplinaire HAL, est destinée au dépôt et à la diffusion de documents scientifiques de niveau recherche, publiés ou non, émanant des établissements d'enseignement et de recherche français ou étrangers, des laboratoires publics ou privés. 


\title{
Résistance du substrat de carbone au silicium fondu dans le procédé RAD
}

\author{
J. Goma, A. Oberlin \\ Laboratoire Marcel Mathieu, ER 131 CNRS, \\ 45046 Orléans Cedex, France \\ E. Kerrand et C. Belouet \\ Laboratoires de Marcoussis, Centre de Recherches de la C.G.E., \\ Route de Nozay, 91460 Marcoussis, France
}

(Reçu le 3 octobre 1983, révisé le 26 décembre, accepté le 5 janvier 1984)

\begin{abstract}
Résumé. - Cet article présente les résultats d'une étude sur les origines de la réactivité des pyrocarbones lamellaires de haute température (PLHT) avec le silicium fondu. Les dépôts de PLHT constituent le revêtement protecteur d'un ruban de graphite souple utilisé comme support dans la croissance de couches minces de silicium par le procédé RAD dans le cadre d'applications photovoltaïques. Ce type de revêtement a été choisi en raison de sa non-réactivité avec le silicium fondu en l'absence de défauts de texture. Les études d'interfaces silicium-pyrocarbone par microscopie électronique en transmission montrent que la réactivité est en relation directe avec la densité de bords libres de couches aromatiques. On montre que dans le cas de conditions de croissance bien contrôlées (dépôt par défilement continu en four ouvert), les PLHT déposés sur un ruban de graphite préalablement purifié au chlore ne présentent plus de défauts de texture. En corollaire, ce type de revêtement évite une contamination du bain de silicium par le ruban de carbone.
\end{abstract}

\begin{abstract}
The results of a study on the origin of the reactivity of high-temperature lamellar pyrocarbons (HTLP) with molten silicon are presented. HTLP layers form the protective coating of the soft graphite ribbon which is used in the RAD process as a substrate for the growth of thin silicon layers in relation to photovoltaic applications. This type of coating was selected for its low reactivity with molten silicon in the absence of textural defects. On the basis of studies of silicon-pyrocarbon interfaces by means of transmission electron microscopy, it is concluded that the reactivity is in direct relation to the density of free edges of aromatic layers. It is shown that HTLP grown on a graphite ribbon previously purified in chlorine do not exhibit textural defects if their deposition conditions are carefully controlled (e.g., by means of a continuous growth process in an open oven). In turn, perfect HTLP coatings obviate the contamination of the melt by the carbon ribbon.
\end{abstract}

\section{Introduction.}

De nombreuses méthodes de croissance de silicium en ruban ont été mises en ouvre depuis une décennie en relation avec le développement de l'énergie photovoltaïque [1]. Parmi celles-ci, le procédé RAD (Ribbon Against Drop) est fondé sur la cristallisation d'un film de silicium liquide sur chaque face d'un ruban de carbone déplacé verticalement à travers un creuset (Fig. 1); les couches de silicium opposées sont ensuite séparées du ruban de 'carbone par combustion de celui-ci à haute température [2].
Ce mode de croissance sur support temporaire présente d'importants avantages dont une capacité de production élevée et l'obtention de dépôts minces, de l'ordre de 50 à $100 \mu \mathrm{m}$, bien adaptés pour la conversion photovoltaïque [3]. Cependant, la présence du ruban de carbone entraîne une contamination du bain de silicium, une limitation de la vitesse de tirage et enfin l'apparition de contraintes thermoélastiques dans les couches de silicium lors du refroidissement. Ces aspects relèvent de l'affinité chimique des carbones avec le silicium fondu et des propriétés thermiques et thermomécaniques du support. 


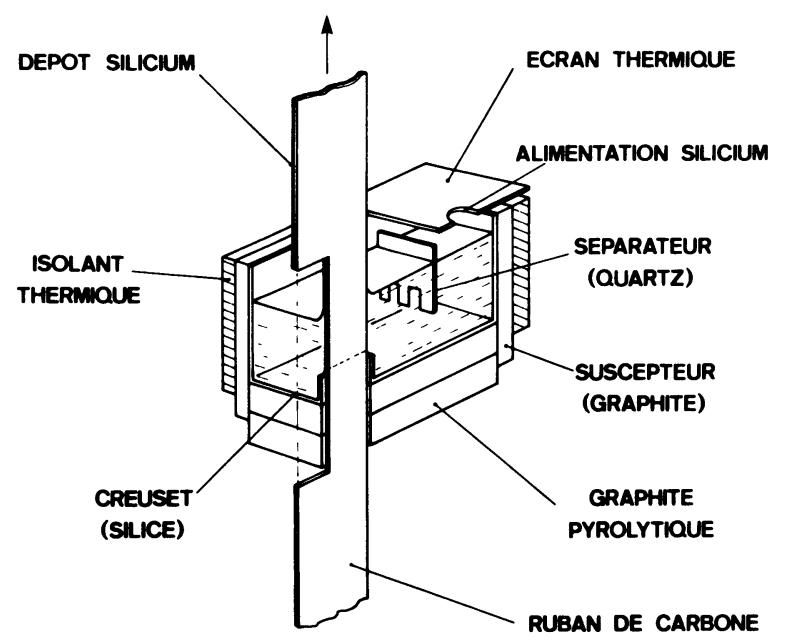

Fig. 1. - Schéma du principe du procédé RAD.

[Sketch of the RAD process.]

La structure de ruban recherchée visait à optimiser séparément les aspects chimiques d'une part, thermiques et thermomécaniques d'autre part, par adaptation des caractéristiques appropriées de l'âme du ruban et de son revêtement.

Cet article traite essentiellement des problèmes de réactivité chimique du support - à base de graphite et de son revêtement - à base de pyrocarbone - avec le silicium fondu. Les méthodes ex 'rimentales utiisees ans cette etude sont décrites d'abord; les propriétés d'un ruban de graphite particulier
(Papyex) $\left({ }^{1}\right)$ sont étudiées ensuite. La réactivité des pyrocarbones est corrélée à leur texture et les imperfections de la variété de pyrocarbone la moins réactive - Pyrocarbone Lamellaire de Haute Température (PLHT) - responsables d'une réactivité résiduelle sont expliquées en termes d'instabilité des conditions de croissance et d'effets des impuretés.

\section{Méthodes expérimentales.}

La réactivité du ruban de graphite et de son revêtement a été étudiée par observation d'interfaces silicium-carbone provenant soit du ruban RAD, soit le plus souvent d'échantillons préparés par la méthode de goutte posée [4]. Celle-ci consiste à déposer une goutte de silicium sur l'échantillon à étudier dans des conditions de température et de durée de contact bien contrôlées, la réaction étant interrompue par solidification rapide du silicium au moyen d'un jet d'hélium (Fig. 2).

Les interfaces silicium-carbone ont été observées au microscope à balayage (MEB) soit sur des coupes transversales, polies, soit sur la face de silicium en regard du carbone après oxydation de celui-ci par voie électrochimique ou par brûlage dans l'oxygène; les échantillons observés en microscopie en transmission (MET) ont été préparés par amincissement ionique [5].

( ${ }^{1}$ ) Nom du ruban de graphite commercialisé par le Carbone Lorraine.

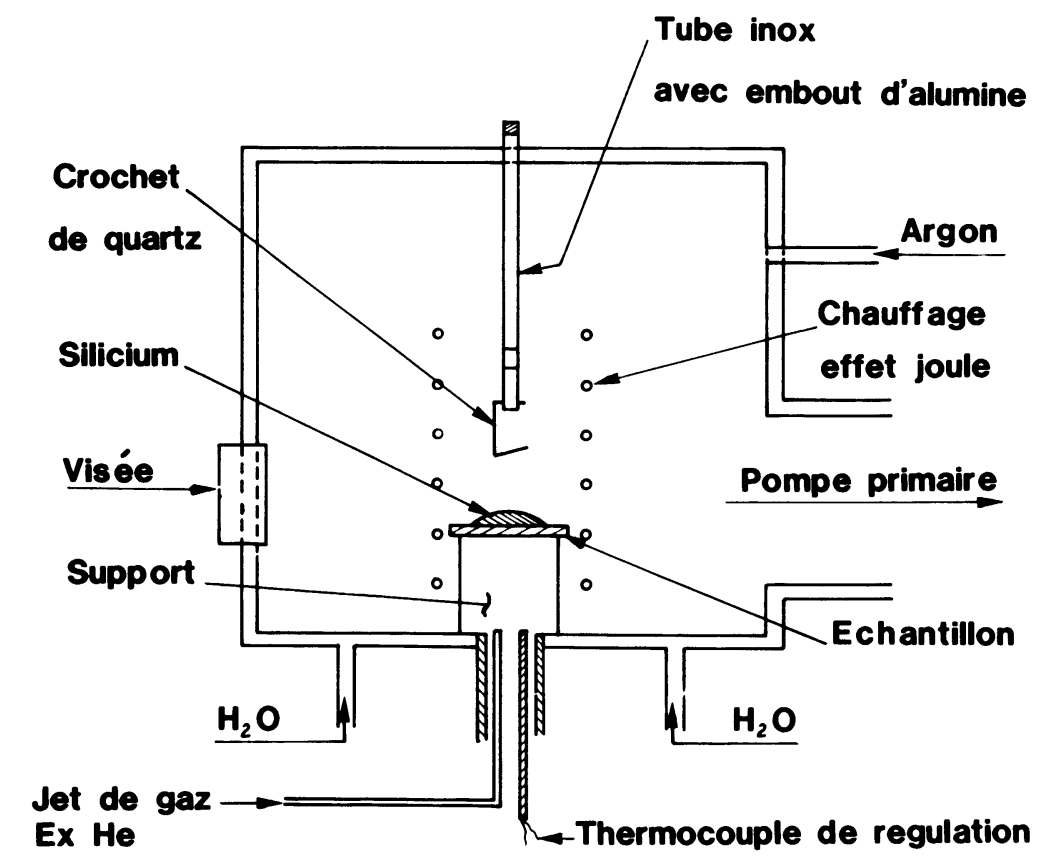

Fig. 2. - Schéma du principe de la goutte posée.

[Sketch of the sessile-drop method.] 
La texture et la structure des pyrocarbones, ainsi que celles du ruban de graphite, ont été étudiées par les techniques de microscopie en transmission [6] fond clair, fond noir, diffraction électronique à aire sélectionnée et visualisation de franges 002 - sur des ézhantillons broyés ou préparés par amincissement ionique. Les analyses de concentration globale d'impuretés ont été faites par une technique d'activation aux neutrons (réacteur OSIRIS de Saclay) [7]; elles ont été complétées par des microanalyses effectuées en spectrométrie de rayons $\mathrm{X}$ dans un microscope électronique à balayage.

\section{Résistance du substrat de carbone au silicium fondu.}

3.1 REMARQUES GÉNÉRALES. - Dans le procédé RAD, le ruban de carbone n'intervient pas directement sur la nucléation du dépôt de silicium [2]; par contre, il influence les échanges de chaleur à l'interface de cristallisation (donnés par la relation 1) dans le cas d'une interface de croissance horizontale, en agissant sur la valeur du gradient de température longitudinal, $G$, dans le solide :

$$
K_{\mathrm{s}} G_{\mathrm{s}}=K_{1} G_{1}+L \rho u .
$$

Dans cette relation, $K, L, \rho$ sont respectivement la conductibilité thermique, la chaleur de cristallisation et la densité du silicium, $G$ est le gradient de température longitudinal, les indices s et 1 se rapportent au solide et au liquide et $u$ est la vitesse de croissance. Ainsi, la vitesse de croissance maximale, $u_{\mathrm{m}}$, donnée par la relation (2) pour $G_{1} \sim 0$ :

$$
u_{\mathrm{m}} \sim K_{\mathrm{s}} G_{\mathrm{s}} / L \rho
$$

est fonction des caractéristiques du ruban de carbone dont dépend $G_{\mathrm{s}}$ [2].

Par ailleurs, la mise en contact du ruban de carbone avec le silicium fondu implique une contamination du bain par le carbone et ses impuretés, notamment métalliques, néfastes pour les performances des photopiles [8].

Enfin, lors du refroidissement du ruban composite après solidification du silicium, les contraintes thermoélastiques induites par le ruban de carbone sont susceptibles de fracturer les dépôts de silicium, si les caractéristiques de ce ruban ne sont pas soigneusement ajustées. L'analyse théorique des sensibilités de la vitesse de tirage, $u_{\mathrm{m}}$, et des contraintes, $\sigma_{(1,3)}\left({ }^{2}\right)$ dans les couches de silicium aux caractéristiques paramétrées du ruban de carbone, tableaux $\mathrm{Ia}, \mathrm{Ib}, \mathrm{a}$ conduit au choix d'un ruban à base de graphite (Papyex) dont les propriétés mécaniques et physiques peuvent permettre d'atteindre les objectifs typiques suivants :

vitesse de tirage, $u_{\mathrm{m}} 10$ à $12 \mathrm{~cm} / \mathrm{min}$,

$\left(^{2}\right)$ Les indices 1 et 3 correspondent aux couches opposées de silicium appelées 1 et 3 .
Tableau Ia. - Domaines du procédé RAD influencés par les paramètres du ruban de carbone.

[RAD process areas influenced by the carbon ribbon properties.]

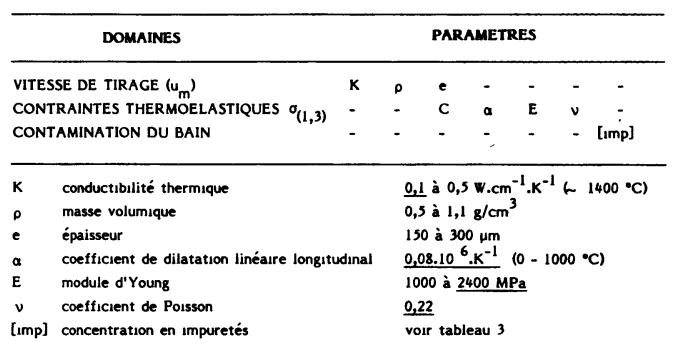

Tableau Ib. - Sensibilités de la vitesse de tirage $u_{\mathrm{m}}$ et des contraintes thermoélastiques $\sigma_{(1,3)}$ dans les couches de silicium (épaisseurs égales à $50 \mu \mathrm{m}$ ). Les calculs ont été effectués avec les valeurs des paramètres soulignées dans le tableau Ia.

[Sensitivities of the pull rate $u_{\mathrm{m}}$ and the thermoelastic stresses $\sigma_{(1,3)}$ in the silicon layers (thicknesses are assumed to be $50 \mu \mathrm{m}$ ). Calculations were made with

\begin{tabular}{|c|c|c|}
\hline \multirow{2}{*}{ PARAMETRES } & \multicolumn{2}{|c|}{ SENSIBILTES" $\sigma, u_{m}$} \\
\hline & $\sigma_{(1,3)}(\mathrm{MPa})$ & $u_{m}(\mathrm{~cm} / \mathrm{mn})$ \\
\hline $\begin{array}{l}\text { Epalsseur pyrocarbone, } e_{0}(\mu \mathrm{m}) \\
\text { Epalsseur Papyex, } e_{2}(\mu \mathrm{m}) \\
\text { Module d'Young Papyex, } E_{2}(\mathrm{MPa}) \\
\text { Masse volumique Papyex }, \rho_{2}\left(\mathrm{~g} / \mathrm{cm}^{3}\right) \\
\text { Conductibulté thermique Papyex, } \mathrm{K}_{2}\left(\mathrm{w} \cdot \mathrm{cm}^{-1} \cdot \mathrm{K}^{-1}\right)\end{array}$ & $\begin{array}{l}\partial \sigma / e_{0} \approx 0,22 \\
\partial \sigma / e_{2} \approx 0,10 \\
\partial \sigma / E_{2} \approx 0,006\end{array}$ & $\begin{array}{l}\partial u_{m} / \partial e_{2}=-1,39 \times 10^{-2} \\
\partial u_{m} / \partial \rho_{2}=-1,2 \\
\partial u_{m} / \partial k_{2}=-11,7\end{array}$ \\
\hline $\begin{array}{l}\text { - DoNNEES: } \\
\text { Eparsseur siltcium, } e_{(1,3)}=50 \mu \mathrm{m}, \mathrm{e}_{2} \\
\text { Module d'Young sllicium, } \mathrm{E}_{(1,3)}=13 \mathrm{C} \\
\text { Domaine de température, } \Delta \mathrm{\Delta T}=100\end{array}$ & $\begin{array}{l}200 \mu \mathrm{m}, \mathrm{e}_{\mathrm{o}}=2 \mu \mathrm{m} \\
100 \mathrm{MPa}, \mathrm{E}_{2}=2400 \\
\mathrm{C}\end{array}$ & $\mathrm{a}, \mathrm{E}_{\mathrm{o}}=15000 \mathrm{MPa}$ \\
\hline
\end{tabular}
the data in table Ia.]

épaisseur de chaque couche de silicium, $e_{(1,3)} 50$ à $100 \mu \mathrm{m}$.

contraintes thermoélastiques résiduelles $\sigma_{(1,3)} \leqslant$ $30 \mathrm{MPa}$.

3.2 Ruban De GRaPhite (PaPyex). - Les rubans du type Papyex sont obtenus par laminage à froid de composés d'insertion du graphite préalablement exfoliés par chauffage brutal vers $1000^{\circ} \mathrm{C}$ [9].

L'observation au microscope électronique en transmission d'échantillons de ce matériau préparés par amincissement ionique permet de décrire la préparation du Papyex suivant le schéma de la figure 3; l'exfoliation du graphite aboutit à la formation de cristallites présentant une texture vermiforme très allongée suivant l'axe c du graphite. Cette texture oriente les axes $\mathbf{c}$ des couches graphitiques parallèlement au plan support (Figs. 3a, 3b). Lors de la compression au laminage, les couches graphitiques se courbent. se chevauchent et s'enchevêtrent (Fig. 3c). A la suite de ce traitement, elles sont donc plissées et entrelacées (Fig. 3d), ce qui confère sa cohésion mécanique au ruban Papyex. 


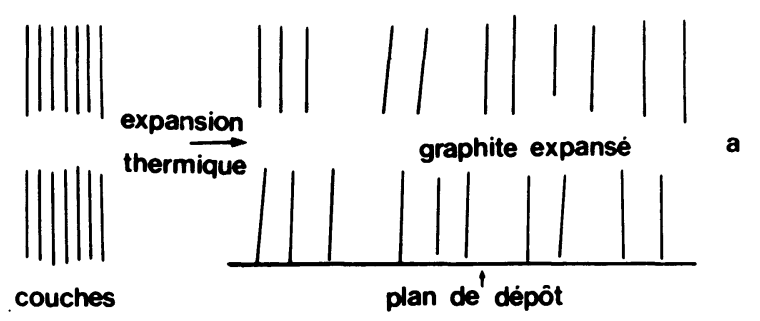

graphitiques
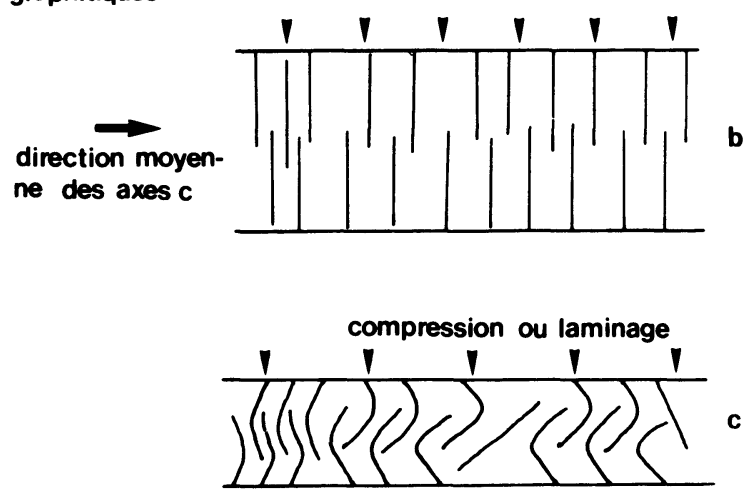

Papyex (LCL)
Grafoil (UC) $\longrightarrow \Longrightarrow C \rightleftharpoons$

Fig. 3. - Processus d'élaboration du Papyex.

[Concept of Papyex ribbon fabrication.]

Ce mode de préparation est à l'origine de la macroporosité importante du Papyex et du nombre élevé de bords de couches graphitiques libres observées en MET (Fig. 4). A plus grande échelle (microscopie optique et MEB), le Papyex se caractérise par son anisotropie due à l'orientation préférentielle des couches graphitiques suivant le plan du ruban.
Mis en présence de silicium fondu, ce matériau réagit très fortement; la réaction se traduit par la formation d'une épaisse couche de carbure de silicium (SiC) dans le réseau du Papyex. Les études en diffraction à aire sélectionnée (DEAS) montrent que cette couche, non continue, est formée de cristaux cubiques (cuboctaèdres) de $\mathrm{SiC}-\beta$ dont une famille de plans (111) est parallèle à la direction moyenne des couches graphitiques du Papyex, (Fig. 5). Ainsi, la direction $\langle 111\rangle$ de SiC- $\beta$ (de symétrie hexagonale suivant cette direction) est parallèle à $\langle 001\rangle$ du graphite. Les sites des atomes de carbone dans les couches carbonées de $\mathrm{SiC}-\beta$ sont répartis aux sommets de triangles équilatéraux de $3,073 \AA$ de côté. On remarque que cette distance est comparable à une des distances entre deux atomes de carbone dans une couche aromatique de graphite (Fig. 6).

D'autre part, la structure du SiC- $\beta$ le long de la rangée $\langle 111\rangle$ est une alternance de plans silicium et carbone, ces derniers occupant les cotes $0, \frac{1}{4}, \frac{3}{4}$ et 1 (Fig. 7). Le paramètre de cette rangée est $7,53 \AA$, distance proche du paramètre c du graphite $(c=6,708 \AA)$. Le réseau du graphite est donc remplacé de façon approchée par le réseau du carbone dans $\mathrm{SiC}-\beta$. Ces observations permettent de conclure que la réaction du silicium liquide avec le ruban de Papyex est une réaction topotactique [10].

Compte tenu de la stabilité chimique des liaisons $\sigma$ dans les couches aromatiques du graphite, la très forte réactivité du ruban Papyex avec le silicium fonđu a été attribuée à la présence de bords de couches libres, très nombreux dans la structure macroporeuse de ce matériau.

Le ruban de Papyex ne pouvant donc intrinsèquement satisfaire au critère de faible réactivité, les études ont été orientées vers la recherche d'une structure constituée par le Papyex recouvert d'une couche mince de pyrocarbone.

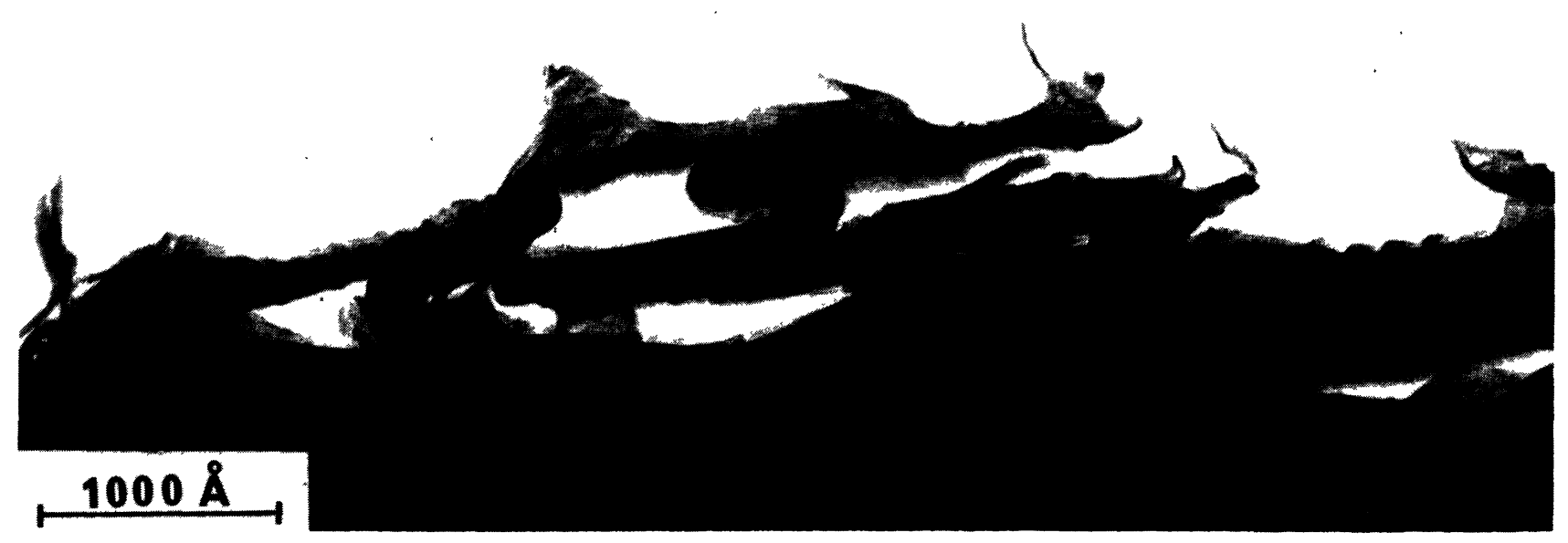

Fig. 4. - Texture du Papyex en MET après amincissement ionique (image en fond clair).

[Papyex texture after ion thinning as observed by TEM (bright field image).] 


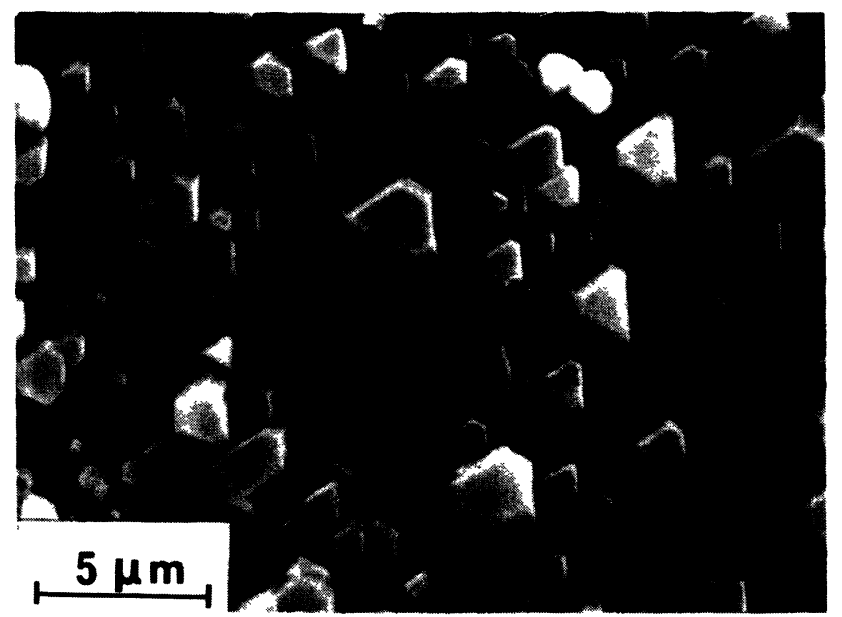

Fig. 5. - Cristaux de SiC- $\beta$ formés par réaction du silicium fondu avec le Papyex (image MEB).

$[\beta$-SiC crystals formed during exposure of Papyex to molten silicon (SEM image).]

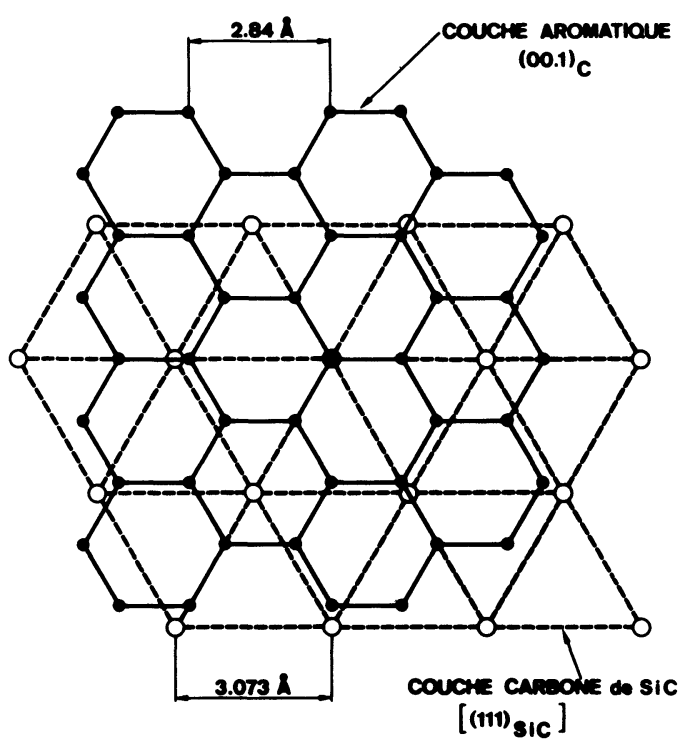

Fig. 6. - Superposition de plans carbonés du SiC- $\beta$ et du graphite.

[Superposition of the carbon planes of silicon carbide and graphite.]

L'étude en microscopie électronique par transmission des textures et de la réactivité des principaux types de pyrocarbones [11] - isotrope, laminaire lisse et lamellaire [12] - a montré que les deux premiers types (laminaire lisse et isotrope) se caractérisent par une porosité importante, ce qui implique une densité de bords de couches libre très élevée, notamment dans le cas des pyrocarbones isotropes. Ils présentent une forte réactivité en présence de silicium fondu (Fig. 8). La réactivité des pyrocarbones isotropes aboutit à la formation de microcristaux

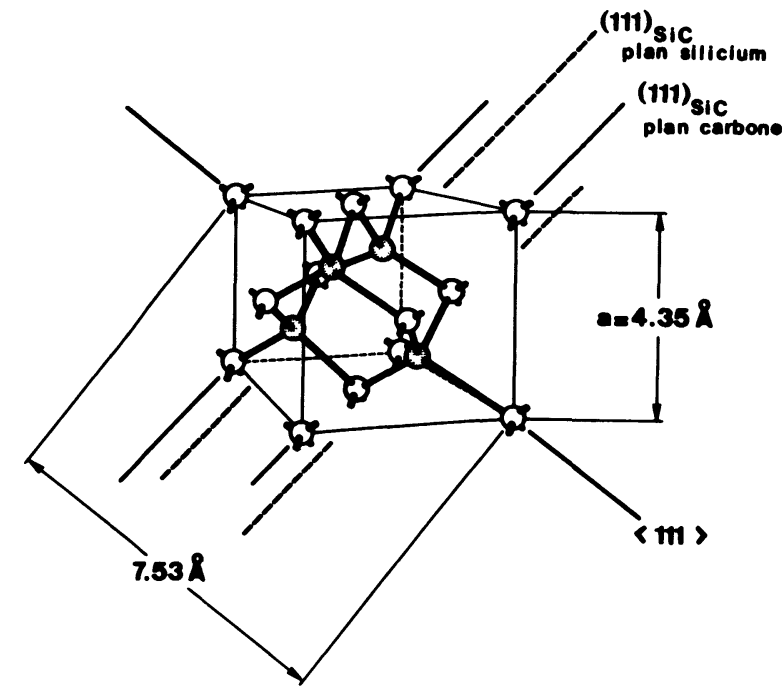

Fig. 7. - Schéma de la maille élémentaire du SiC- $\beta$.

[Sketch of the $\beta$-SiC unit cell.]

de SiC- $\beta$ (Fig. 8a) tandis que les pyrocarbones laminaires lisses présentent une réactivité plus ou moins importante en relation avec la densité locale de pores (Fig. 8b). Cette réactivité des pyrocarbones isotrope et laminaire lisse a permis de confirmer l'hypothèse de l'attaque des carbones par les bords de couches libres et de retenir un pyrocarbone lamellaire de haute température comme revêtement de Papyex.

\subsection{Pyrocarbone lamellaire de haUte tempéra-} TURE. - Les dépôts de pyrocarbone sur le ruban de Papyex ont été réalisés en four ouvert par décomposition pyrolytique de méthane à $2100^{\circ} \mathrm{C}$ en présence d'hydrogène et sous une pression de quelques torrs [13].

Les PLHT ainsi obtenus se caractérisent par une structure compacte illustrée par la figure 9 (micrographie MET). Les couches aromatiques y sont orientées parallèlement au plan de support (ici la surface du ruban de graphite). Des observations effectuées sur des échantillons broyés montrent que ces pyrocarbones sont formés de lamelles étendues (Fig. 10) dont le plan est parallèle au support.

Du fait de leur structure lamellaire, ces pyrocarbones ne présentent pas de bords de couches libres. Il en résulte une absence de réactivité avec le silicium fondu $[14,15]$ illustrée par la figure 11 : celle-ci montre l'interface silicium-substrat au niveau d'une fracture du revêtement de pyrocarbone. Au contraire du ruban de Papyex (voir Fig. 5), le revêtement de pyrocarbone lamellaire est resté inaltéré sur chacune de ses faces hors du voisinage immédiat de la fracture après exposition au silicium fondu; on remarque cependant qu'une importante formation de $\mathrm{SiC}$ s'est propagée, à partir des bords de la fracture dans le dépôt de pyrocarbone. Cette anisotropie de réactivité est en relation directe avec la structure des pyrocarbones lamellaires. 


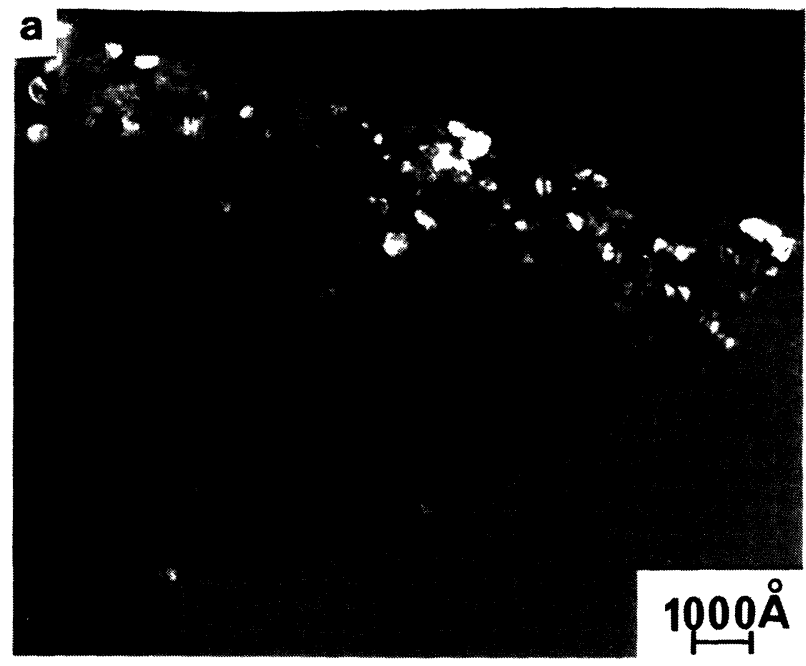

b

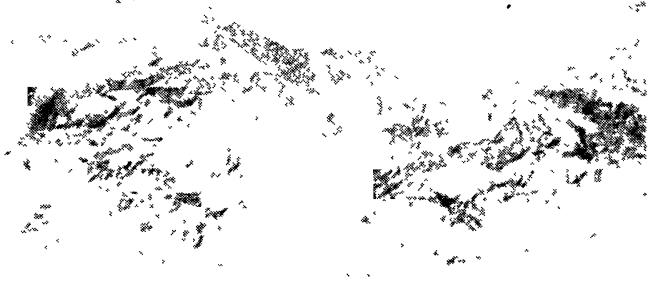

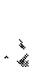

$1000 \AA$

Fig. 8. - Réactivité de pyrocarbones poreux en présence de silicium fondu : a) pyrocarbone isotrope (microporeux); b) pyrocarbone laminaire lisse (poreux orienté d'après J. Goma et al. [14].

[Reactivity of porous pyrocarbons with molten silicon : a) isotropic pyrocarbon (microporous); b) smooth laminar pyrocarbon (flattened pores) from J. Goma et al. [14].]

La résistance remarquable des pyrocarbones lamellaires de haute température au silicium fondu, dans les conditions du procédé RAD, est indépendante de l'épaisseur " $e_{0}$ " du dépôt dans la gamme de 2 à $50 \mu \mathrm{m}$ étudiée. Ainsi, compte tenu de la vitesse de croissance de ces pyrocarbones $(\simeq 1 \mu \mathrm{m} / \mathrm{min})$, le revêtement peut être réalisé en four ouvert à des vitesses de défilement relativement élevées [13]. Par ailleurs, pour des valeurs de $e_{0}$ de l'ordre de quelques microns, la présence de ce revêtement n'influe pas significativement sur les valeurs de $u_{\mathrm{m}}$ et $\sigma$; ce dernier cas est illustré dans le tableau I qui donne les sensibilités de $\sigma$ aux paramètres appropriés du ruban de carbone et les caractéristiques physiques du ruban type actuel pour lequel $\sigma$ est de l'ordre de $30 \mathrm{MPa}$ [16].

Cependant, les dépôts PLHT en couches minces peuvent présenter un certain nombre d'imperfections à l'origine d'une réactivité résiduelle.

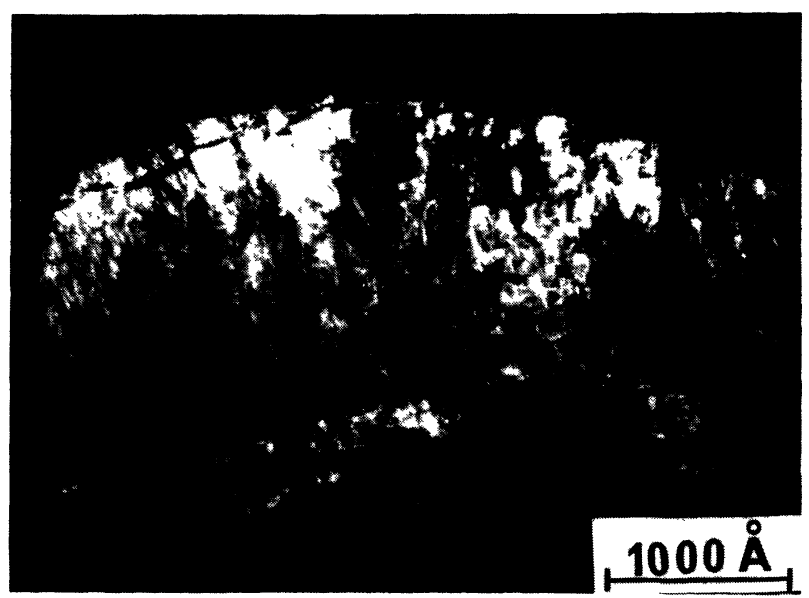

Fig. 9. - Pyrocarbone lamellaire de haute température après amincissement ionique (image en fond noir 002).

[High-temperature lamellar pyrocarbon (HTLP) after ion thinning as observed by CTEM (002 dark field image).]

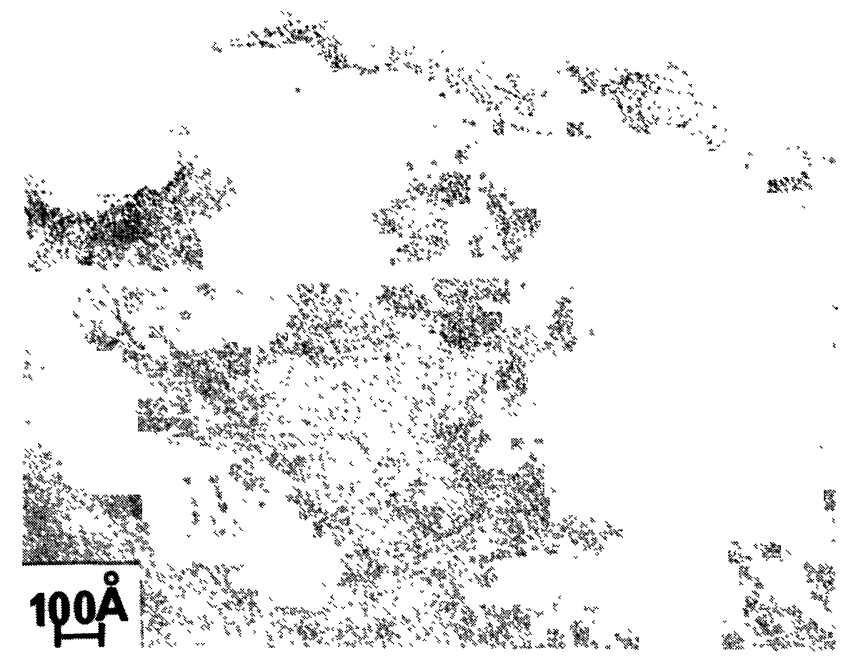

Fig. 10. - Lamelles de PLHT observées en MET sur un échantillon broyé (image en fond clair).

[Bright-field image (CTEM) of HTLP lamellar after grinding.]

\section{Imperfections des revêtements PLHT.}

Les imperfections rencontrées ont généralement pu être attribuées aux instabilités des conditions de croissance avec formation de noirs de carbone et à la présence d'impuretés métalliques provenant de l'enceinte de dépôt ou du substrat.

4.1 NoIrS DE CARBONE. - Les noirs de carbone sont des particules sphériques qui possèdent une texture en pelure d'oignon où toutes les couches aromatiques (formant de petits empilements turbostratiques d'environ $15 \AA$ de diamètre) sont orientées 


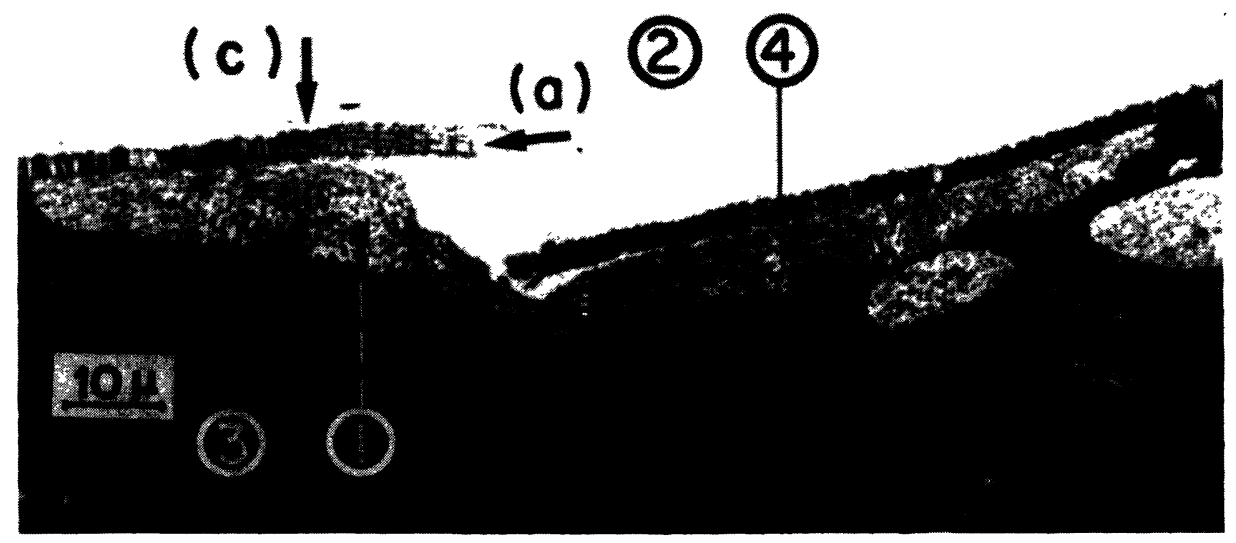

Fig. 11. - Micrographie optique d'une coupe verticale d'un ruban RAD prise au voisinage d'une fracture du revêtement de pyrocarbone. On observe (i) une importante formation de $\mathrm{SiC}$ (1) où le silicium (2) est entré en contact direct avec le substrat de Papyex (3) et (ii) une anisotropie d'attaque du pyrocarbone (4) dans les directions A et B.

[Optical micrograph of a vertical cross-section of an RAD ribbon taken in the vicinity of a crack in the pyrocarbon coating. Observe (i) the large SiC formation (1) when silicon (2) was exposed to the Papyex substrate (3); (ii) the anisotropy of the attacks on pyrocarbon (4) in directions $A$ and B.]

concentriquement, parallèlement à la surface de la sphère (Fig. 12). Ils sont issus d'une décomposition thermique ou d'une combustion incomplète d'hydrocarbures $[17,18]$ et sont essentiellement formés de carbone. Leur formation, soit au sein des lamelles de PLHT, soit à la surface de celles-ci, témoigne de variations des paramètres de croissance.

Les noirs de carbone interagissent avec le silicium fondu à deux niveaux : d'une part, ils induisent une importante pollution du silicium en carbone (lorsqu'ils se " dissolvent " dans le bain) et d'autre part, leur départ de la couche mince de pyrocarbone fait apparaître des bords libres par lesquels le silicium peut attaquer le revêtement du Papyex.

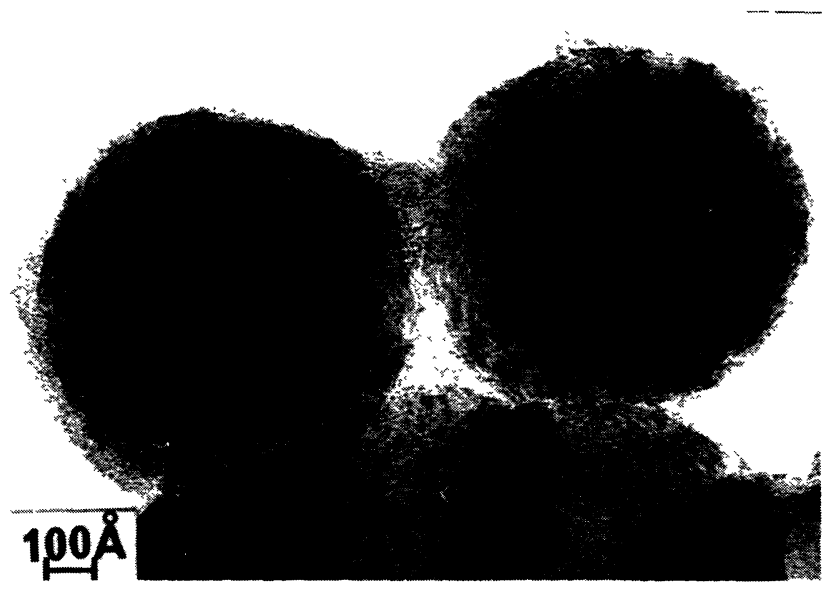

Fig. 12. - Noirs de carbone observés au MET (image en fond clair).

[Bright-field image of carbon blacks (CTEM).]
4.2 EFFETS D'IMPURETÉs. - L'interaction de certaines impuretés $(\mathrm{Fe}, \mathrm{Ni}, \mathrm{Mo} .$.$) avec le gaz carboné est$ susceptible de détruire localement la structure lamellaire des PLHT, du fait de la formation de coques de carbone autour de ces particules [19]. Dans les conditions de dépôt des PLHT, certaines de ces impuretés se vaporisent, laissant une enveloppe de carbone vide. Ces coques, relativement fines, épousent le contour de la particule (à l'origine de leur formation) dont elles conservent l'empreinte. Elles ne sont observables qu'en fond noir ou en visualisation de plans réticulaires (Fig. 13). Leur présence, à la surface des couches

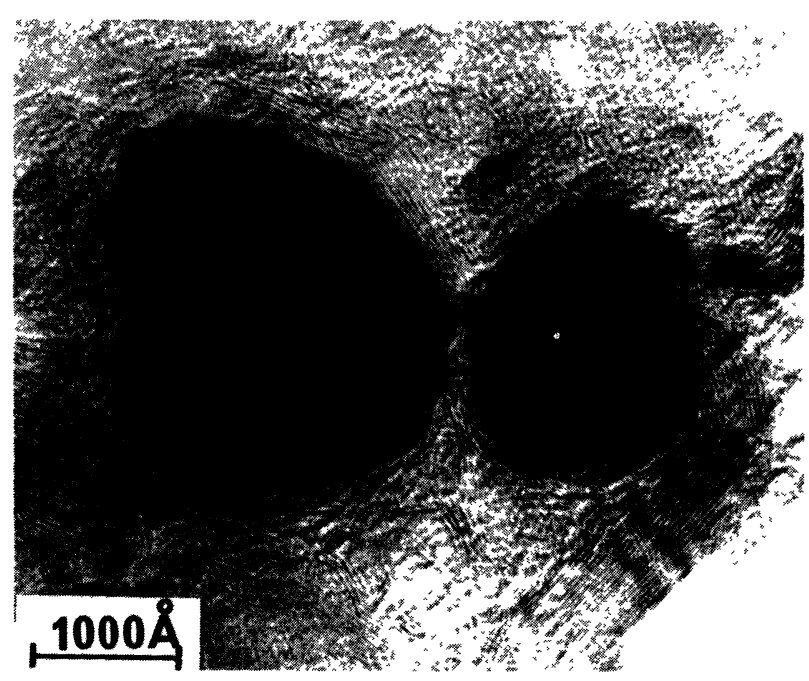

Fig. 13. - Coques de carbone autour de particules métalliques en visualisation de franges 002 .

[002 lattice fringes of carbon shells around metallic particles (CTEM). ] 
minces de PLHT, introduit de nombreuses parois verticales (bords de couches libres) responsables d'une réactivité avec le silicium fondu qui peut être importante.

Outre cette croissance catalytique, d'autres observations ont mis en évidence des interactions impuretés-carbone aux stades du dépôt de silicium et du brûlage du ruban de carbone effectué sous oxygène à $1000^{\circ} \mathrm{C}$, (Figs. 14, 15). La figure 14 montre une croissance de type filamentaire à l'intérieur d'une cavité localisée dans le silicium à l'interface silicium-carbone; une microanalyse de cette zone par spectrométrie $\mathrm{X}$ y a révélé la présence de fer et de nickel. Ces observations suggèrent un mécanisme de croissance

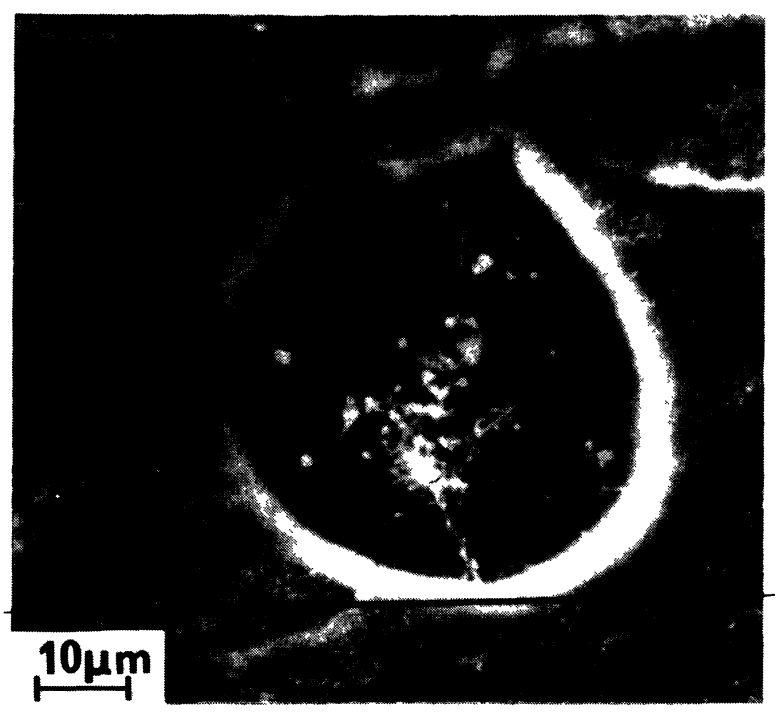

Fig. 14. - Croissance filamentaire de carbone catalysée par le fer et le nickel.

[Carbon filament growth catalyzed by $\mathrm{Fe}$ and Ni.]

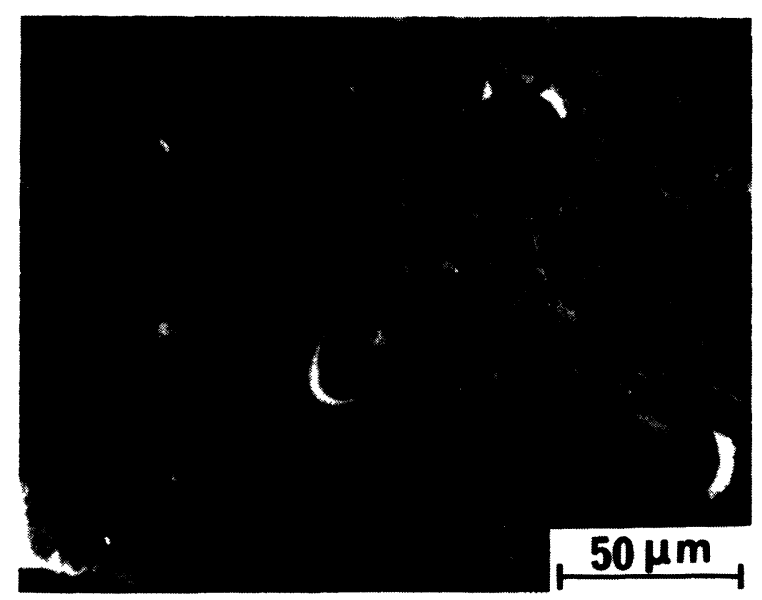

Fig. 15. - Canalisations dues à l'activité catalytique d'une particule métallique $(\mathrm{Cu})$ à l'interface silicium-carbone.

[Channelling due to the catalytic activity of a metallic particle $(\mathrm{Cu})$ at the silicon-carbon interface.] de carbone filamentaire du type décrit par Audier et al. qui implique un transport en phase vapeur par l'oxyde de carbone (CO) [20].

Cet oxyde de carbone est responsable d'une formation de $\mathrm{SiC}-\alpha$ syncristallisé avec le silicium à l'interface silicium-carbone dont l'épaisseur peut être supérieure d'au moins un ordre de grandeur à celle simplement déduite du diagramme de phase silicium-carbone [15]. La présence de cet oxyde est imputable à l'oxygène adsorbé dans le ruban de carbone conservé au contact de l'air et à la réaction de l'oxygène de contamination de la chambre de croissance du four en graphite (voir Fig. 1). La figure 15 présente un phénomène de canalisation à l'interface silicium-carbone [21]. Cette activité catalytique, reliée par ailleurs à l'opération de brûlage, se caractérise par la formation de canaux vides à l'extrémité desquels se trouve une particule métallique (cuivre dans le cas présent).

Le tableau III donne les concentrations des impuretés dans le ruban de carbone à ses différents stades de fabrication [22]. Les teneurs des éléments As, Au, $\mathrm{Br}, \mathrm{Cd}, \mathrm{Ga}, \mathrm{K}$ et $\mathrm{Sb}$, non présentés dans ce tableau, étaient inférieures à $0,1 \mu \mathrm{g} / \mathrm{g}$.

Tableau II. - Effets d'impuretés dans le procédé RAD.

[Impurity effects in the RAD process.]

\begin{tabular}{|c|c|c|c|c|}
\hline IMPURETES & $\begin{array}{c}\text { MILIEUX } \\
\text { d' INTE RACTION } \\
\end{array}$ & ETAPES & REACTIONS & FORMATIONS \\
\hline $\mathrm{Fe}, \mathrm{N}_{1}, \mathrm{Mo} \ldots$ & c & dépôt pyrocarb. & catalyse [19] & coques (fig. 13) \\
\hline $\mathrm{Fe}, \mathrm{N}_{\mathrm{t}}$ & $\mathrm{co}^{*}$ & dépôt silicium & $\begin{array}{l}\text { croissance } \\
\text { filamentaire }\end{array}$ & filaments (fig. 14) \\
\hline $\mathrm{Cu}$ & $\mathrm{CO}+\mathrm{C}$ & $\begin{array}{c}\text { séparation } \\
\text { (brûlage) }\end{array}$ & canalisation [21] & canaux' (fig. 15) \\
\hline $\mathrm{CO}^{*}$ & $s_{1}$ & dépôt sılicium & dissolution [14] & $\mathrm{S} \mathrm{C}-\alpha$ \\
\hline
\end{tabular}

Tableau III. - Analyse par activation neutronique du ruban de carbone (concentration en $\mu \mathrm{g} / \mathrm{g}$ ).

[Neutron activation analysis of the carbon ribbons (impurity content $: \mu \mathrm{g} / \mathrm{g}$ ).]

\begin{tabular}{|c|c|c|c|c|c|}
\hline \multirow{2}{*}{ ELEMENTS } & \multirow{2}{*}{$\begin{array}{l}\text { PAPYEX } \\
\text { BRUT }\end{array}$} & \multirow{2}{*}{$\begin{array}{l}\text { PAPYEX } \\
\text { PURIFIE (D) }\end{array}$} & \multirow{2}{*}{$\begin{array}{l}\text { PAPYEX } \\
\text { PURIFIE (S) }\end{array}$} & \multicolumn{2}{|c|}{ RUBAN DE CARBONE * } \\
\hline & & & & $\overline{\text { essai } A}$ & essat B \\
\hline $\mathrm{Cl}$ & 26,3 & 14,0 & 6,0 & 5,0 & 7,6 \\
\hline Co & 1,5 & $5,0 \times 10^{-3}$ & $3,5 \times 10^{-3}$ & $3,0 \times 10^{-1}$ & $4,1 \times 10^{-3}$ \\
\hline $\mathrm{Cr}$ & 39,8 & $6,0 \times 10^{-2}$ & $4,0 \times 10^{-2}$ & 10 & $6,6 \times 10^{-2}$ \\
\hline $\mathrm{Cu}$ & 20,3 & $9,0 \times 10^{-2}$ & $3,0 \times 10^{-1}$ & $1,0 \times 10^{-1}$ & $2,4 \times 10^{-1}$ \\
\hline $\mathrm{Fe}$ & 70,5 & $8,0 \times 10^{-1}$ & 1,5 & 65,5 & 1,3 \\
\hline Mn & 8,4 & $7,0 \times 10^{-3}$ & $7,7 \times 10^{-3}$ & $1,0 \times 10^{-2}$ & $1,5 \times 10^{-2}$ \\
\hline Mo & 100,0 & 7,3 & 2,5 & 36,7 & 1,6 \\
\hline $\mathrm{Na}$ & 16,0 & $7,0 \times 10^{-1}$ & 3,9 & 1,0 & $\sim 1$ \\
\hline $\mathrm{N}_{1}$ & 62,0 & $<3,0 \times 10^{-1}$ & $\left\langle 5,0 \times 10^{-1}\right.$ & 2,6 & $4,2 \times 10^{-1}$ \\
\hline w & 5,0 & $5,0 \times 10^{-2}$ & $8,0 \times 10^{-2}$ & 1,8 & $8,4 \times 10^{-2}$ \\
\hline$z_{n}$ & 170,0 & $1,0 \times 10^{-1}$ & $5,0 \times 10^{-1}$ & $<2,0 \times 10^{-1}$ & $2,9 \times 10^{-1}$ \\
\hline
\end{tabular}

(D) Purification sous chlore en dynamıque à $2000^{\circ} \mathrm{C}$

(S) Purification sous chlore en statique à $2800^{\circ} \mathrm{C}$.

"Les dépôts de pyrocarbone ont été effectués sur ruban de Papyex brut (essaı A) et purifié au chlore en dynamıque (essa! B). 
On remarque que les impuretés du ruban complet sont essentiellement celles du Papyex de départ très contaminé en impuretés métalliques dont $\mathrm{Fe}, \mathrm{Mo}, \mathrm{Ni}$, $\mathrm{Zr}$..., par ailleurs néfastes pour les performances des photopiles. Les études des interactions impuretéscarbone ci-dessus suggèrent qu'une partie au moins de ces impuretés se présentent en amas; ceci a été confirmé par des observations MEB sur des échantillons préalablement oxydés sous atmosphère d'oxygène vers $800^{\circ} \mathrm{C}$ (Fig. 16). Le tableau II résume les conséquences générales des effets d'impuretés dans le procédé RAD.

Le rôle important joué par les impuretés du ruban de graphite, notamment au niveau de la texture du PLHT, a motivé une étude de purification de ce dernier. Cette purification a été réalisée par traitement en atmosphère de chlore à haute température, soit en dynamique (D) dans un four à défilement à $2100^{\circ} \mathrm{C}$, soit en statique (S) à $2800^{\circ} \mathrm{C}$ [23].

Les résultats du tableau III montrent l'efficacité de ce traitement pour la plupart des impuretés, chlore et sodium exceptés, le facteur de purification variant de 10 à $10^{3}$; on remarque cependant que la concentration en molybdène reste élevée compte tenu de son influence sur les performances des photopiles. La croissance catalytique dans les dépôts PLHT réalisés sur des rubans de graphite préalablement purifiés (du type " $D$ » ou " $S$ » du Tableau III) devient négligeable et, dans ces conditions, on peut obtenir des lamelles parfaites de très grande étendue (voir Fig. 10). De tels revêtements sont alors inertes vis-à-vis du silicium fondu dans les conditions du procédé RAD; en corollaire, la contamination des bains de silicium au contact de tels rubans est relativement faible, à l'exception du molybdène. Ceci apparaît dans le tableau IV qui donne les teneurs en impuretés dans le silicium de départ et dans les bains exposés à des rubans purifiés (B) et non purifiés $(\mathrm{A})$; on a aussi mentionné dans ce tableau l'effet de ségrégation au front de cristallisation dans le cas de l'essai A.

L'élimination des effets d'impuretés ci-dessus peut donc être obtenue par un traitement chlore du ruban de graphite de départ et un conditionnement du ruban recouvert de pyrocarbone sanś remise à l'air avant le dépôt de silicium pour éviter la formation ultérieure d'oxyde de carbone.

Enfin, on notera que la morphologie de surface du ruban Papyex n'intervient pas sur la texture et par conséquent sur la réactivité des PLHT [4].

\section{Conclusions.}

La cristallisation de films de silicium sur ruban de carbone pour applications photovoltaïques impliquait une contamination minimale du silicium par le carbone et la compatibilité des propriétés physiques du ruban de carbone avec les critères de vitesse de tirage élevée et de faibles contraintes thermoélastiques au refroidissement.

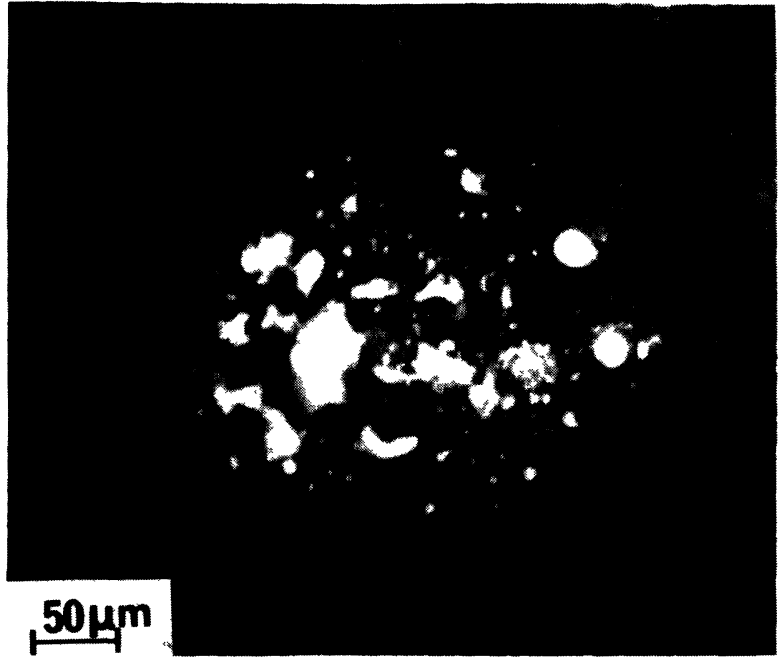

Fig. 16. - Amas d'impuretés révélées après oxydation du ruban (Papyex recouvert de pyrocarbone).

[Impurity agglomerates revealed after oxidation of the ribbon (pyrocarbon coated Papyex).]

Tableau IV. - Analyse par activation neutronique du silicium (concentration en atomes $/ \mathrm{cm}^{3}$ ).

[Neutron activation analysis of silicon (impurity content $: \mu \mathrm{g} / \mathrm{g})$.]

\begin{tabular}{|c|c|c|c|c|}
\hline \multirow{2}{*}{ ELEMENTS } & \multirow{2}{*}{ SILICIUM DEPART } & \multicolumn{2}{|c|}{ SILICIUM ESSAI A } & \multirow{2}{*}{$\begin{array}{l}\text { SILICIUM } \\
\text { ESSAI B } \\
\text { BAIN }\end{array}$} \\
\hline & & BAIN & COUCHE & \\
\hline Au & $2,0 \times 10^{10}$ & $7,0 \times 10^{12}$ & $7,0 \times 10^{11}$ & $4 \times 10^{12}$ \\
\hline Co & $<1,0 \times 10^{13}$ & $5,0 \times 10^{14}$ & $2,4 \times 10^{13}$ & $1 \times 10^{13}$ \\
\hline $\mathrm{Cr}$ & $<5,0 \times 10^{13}$ & $5,0 \times 10^{15}$ & $2,0 \times 10^{15}$ & $<1 \times 10^{13}$ \\
\hline $\mathrm{Cu}$ & $<4,0 \times 10^{13}$ & $5,0 \times 10^{15}$ & $4,0 \times 10^{13}$ & $<4 \times 10^{13}$ \\
\hline $\mathrm{Fe}$ & $<1,5 \times 10^{15}$ & $2,8 \times 10^{17}$ & $<5,0 \times 10^{15}$ & $<2 \times 10^{15}$ \\
\hline Mo & $<9,0 \times 10^{13}$ & $2,2 \times 10^{15}$ & $2,6 \times 10^{14}$ & $1 \times 10^{15}$ \\
\hline $\mathrm{Na}$ & - & $6,0 \times 10^{14}$ & $1,2 \times 10^{14}$ & $2 \times 10^{14}$ \\
\hline $\mathbf{N}_{1}$ & $<1,0 \times 10^{15}$ & $2,0 \times 10^{16}$ & $<5,0 \times 10^{14}$ & $<2 \times 10^{15}$ \\
\hline Sb & $<1,0 \times 10^{12}$ & $9,0 \times 10^{14}$ & $1,2 \times 10^{12}$ & $1 \times 10^{13}$ \\
\hline w & $<5,0 \times 10^{10}$ & $3,8 \times 10^{14}$ & $<5,0 \times 10^{11}$ & $<1 \times 10^{12}$ \\
\hline $\mathrm{zn}$ & $<1,0 \times 10^{14}$ & $1,0 \times 10^{15}$ & $<2,0 \times 10^{14}$ & $<1 \times 10^{14}$ \\
\hline
\end{tabular}

Cet article montre que la structure du ruban de carbone répondant à ces propriétés est constituée d'un ruban de graphite souple (Papyex) recouvert d'un film mince de pyrocarbone lamellaire de haute température (PLHT), qui confère au ruban sa résistance au silicium fondu. Ce type de revêtement a été choisi sur la base d'études de microtextures de pyrocarbones par microscopie électronique en transmission couplées à des tests de réactivité, qui ont permis d'établir une relation directe entre la réactivité des pyrocarbones et leur densité de bords libres de couches aromatiques. Dans des conditions de croissance et de pureté du ruban de graphite support bien déterminées, les PLHT constitués de grandes lamelles graphitiques parallèles au support sont exempts de bords de couche aromatiques et donc non réactifs vis-à-vis du silicium fondu. Le contrôle de la pureté chimique du support de graphite a été obtenu par purification au chlore à haute température. 
Une autre propriété remarquable de ces pyrocarbones est leur vitesse de croissance rapide, compatible avec un dépôt continu en four à défilement. De plus, leur texture est insensible à la morphologie du support et à l'épaisseur du dépôt.

Ainsi, le revêtement de PLHT n'altère pas les caractéristiques mécaniques et thermomécaniques du ruban de carbone. Les performances photovoltaïques des photopiles préparées avec les dépôts de silicium RAD sont similaires à celles obtenues avec des techno- logies en ruban auto-supporté; des rendements de conversion $\mathrm{AM}_{1}$ supérieurs à $13 \%$ ont été obtenus.

\section{Remerciements.}

Les auteurs remercient toutes les personnes qui ont contribué à ce programme. l'Agence Française pour la Maîtrise de l'Energie et l'Electricité de France qui ont financé en partie ces recherches.

\section{Bibliographie}

[1] Consulter par exemple : Shaped Crystal Growth, ed. G. W. Cullen, T. Surek, P. I. Antonov (NorthHolland Publishing Company-Amsterdam) 1980.

[2] Belouet, C., Texier-Hervo, C., Mautref, M., Belin, C., Paulin, J., Schneider, J., J. Crystal Growth 61 (1983) 615.

[3] Hovel, H. J., dans : Semiconductors and Semimetals, Vol. II, Solar Cells (Academic Press), p. 100.

[4] Kerrand, E., Etude de revêtements de pyrocarbones en relation avec leur utilisation comme support de dépôt de film de silicium polycristallin par la méthode RAD. Thèse de $3^{e}$ Cycle (1982), Université Pierre et Marie Curie.

[5] Maissel, L. I., Physics of Thin Films 3 (1966) 61, ed. G. Hass \& R. E. Thun (Academic Press, New York).

BERLIN, ., Carbon 17 (1979) 7.

[7] Revel, G., Fedoroff, M., Nuclear Instrum. Met. 143 (1977) 277.

[8] Seidensticker, R. G., Hopkins, R. H., Davis, J. R., Rai Choudhury, P., Blais, P. D., J. Crystal growth 42 (1977) 493.

[9] MaIre, J., Chim. Ind. 105 (1972) 23.

[10] Bernal, J. D., Schweiz. Arch. (1960) 69 ;

Wyart, J. et Sabatier, G., Bull. Soc. Min. Cryst. 81 (1958) 223.

[11] Goma, J., Pyrocarbones et interfaces silicium-carbone, Thèse d'Etat (1983), Université d'Orléans.

[12] Loll, P., Delhaes, P., Pacault, A. and Pierre, A., Carbon 15 (1977) 363.

[13] Maire, J., Slonina, J. P., Gellon, J., dans : Preprints
« Carbon 80 ", Baden-Baden (Bad-Honnef : Deutsche Keramische Gesell Schaft) 667.

[14] Goma, J., Oberlin, M., Oberlin, A., Schneider, J., Belouet, C., High Temp. High Press. 13 (1981) 263.

[15] Goma, J., Oberlin, M. and Oberlin, A., Revue Phys. Appl. 15 (1980) 229.

[16] Belouet, C., Growth of Silicon Ribbons, à paraitre dans "Silicon Processing for Photovoltaics " (North Holland Publishing Company).

[17] Donnet, J. B., Les Carbones (Paris, Masson \& Cie) 2 (1965) 690.

[18] Lahaye, J., Prado, G., Chem. Phys. Carbon 14 (1978) 167, Ed. P. L. Walker Jr. (Marcel Dekker, New York).

dismutation catalytique de CO. Application $\stackrel{\bar{p} a r}{a}$ synthèse des hydrocarbures à partir de $\mathrm{CO}$ et $\mathrm{H}_{2}$. Thèse d'Etat (1980), Université de Grenoble.

[20] Audier, M., Oberlin, A., Oberlin, M., Coulon, M. et Bonnetain, L., Carbon 19 (1981) 217.

[21] Hennig, G. R., Chem. Phys. Carbon 2 (1966) 1, Ed. P. L. Walker Jr. (Marcel Dekker, New York).

[22] Revel, G., Deschamps, N., Deville, J. P., TexierHervo, C., Belouet, C., dans : Proc. 4th Photovoltaic Solar Energy Conference, STRESA 1982 (Ed. W. H. Bloss et G. Grassi, D. Reidel Publishing Company), p. 970.

[23] Maire, J., Fourre, Gremion, R., Le Carbone Lorraine, 1981, Communication privée. 